Revista de la Facultad de Ingeniería Industrial

15(2): 85-91 (2013) UNMSM

ISSN: 1560-9146 (Impreso) / ISSN: 1810-9993 (Electrónico)

\title{
El maíz morado como materia prima industrial
}

\section{RESUMEN}

Actualmente, el maíz morado es utilizado en forma integral, es decir sin separar los granos del marlo, lo cual constituye un uso irracional, debido a que se descarta los granos, los cuales constituyen alimentos de alto valor nutritivo. Motivado por esta situación, el presente estudio tiene por objetivo el procesamiento del maíz morado, para obtener productos de uso industrial. Estos productos del maíz morado procesado son: marlo triturado, harina y afrecho. El marlo triturado se utiliza para obtener solución acuosa, comúnmente llamado "chicha morada, que también se utiliza para preparar mazamorra morada". La harina puede utilizarse en panificación, y el afrecho como alimento para animales.

Palabras clave: maíz morado, panoja, marlo, antocianina, harina, afrecho.

PurPle CORN AS Industrial RaW Material

\section{ABSTRACT}

Currently, the purple corn is used in integral form, ie without separating grains cob, which is an unreasonable use, because it is discarded grains, which are highly nutritious foods. Motivated by this background, this study aims purple corn processing, products for industrial use. These purple corn processed products are: cob grits, flour and bran. The crushed cob is used to obtain an aqueous solution, commonly called "chicha morada, which is also used to prepare purple porridge". The flour can be used in baking, and bran for animal feed.

Keywords: Purple corn cob, cob, anthocyanin, flour, bran.

\section{INTRODUCCIÓN}

La mazorca o panoja del maíz morado está constituida por granos y marlo o coronta, en una proporción promedio en masa de $80 \%$ de granos y $20 \%$ de marlo. La principal utilidad del maíz morado se debe a su propiedad colorante o tintórea, cuyo poder o capacidad de coloración se encuentra mayoritariamente concentrada en el marlo. Químicamente, la materia colorante del maíz morado es la antocianina, que son glucósidos que se encuentran constituyendo el principio colorante responsable de los colores rojo, violeta, azul y púrpura que aparecen en las flores, frutos, hojas y otros tejidos de las plantas. En el caso del maíz morado la coloración púrpura o morada se encuentra en el marlo y en el pericarpio de los granos [1].

\section{PLANTEAMIENTO DEL ESTUDIO}

La finalidad del estudio es el procesamiento del maíz morado para la obtención de marlo triturado o en polvo y harina. Como subproducto de la harina se obtiene afrecho. El maíz que se va a procesar debe estar en buen estado con un contenido de humedad promedio de $14 \%$.

\section{INFORMACIÓN GENERAL SOBRE LA MATERIA PRIMA}

\section{Clasificación botánica}

El maíz morado pertenece a la familia de las gramíneas, género Zea y especie Zea Mays.

\section{Cuadro comparativo de composición química}

El análisis corresponde a una muestra de $100 \mathrm{~g}$, realizada a tres variedades.

\footnotetext{
* Ing. Químico. Docente Principal de la FII-UNMSM. E-mail: mooner.lavado@unmsm.edu.pe

** Ing. Industrial. Docente Principal de la FII-UNMSM. E-mail: 1raezg@unmsm.edu.pe

*** Ing. Químico. Docente Auxiliar de la FQIQ-UNMSM. E-mail: roberto-r@hotmail.com
} 
Cuadro 1. Composición química de variedades de maíz

\begin{tabular}{|c|c|c|c|c|}
\hline Variedad & Energía (kJ) & Proteínas(g) & Carbohidratos(g) & Vitamina C(mg) \\
\hline Amarillo & 1515 & 8.4 & 69.4 & -- \\
Blanco & 1456 & 5.6 & 74.3 & -- \\
Morado & 1494 & 7.3 & 76.2 & 2.1 \\
\hline
\end{tabular}

Fuente: Editorial Isabel, Tabla de Composición Química de los Alimentos.

\section{PRUEBAS EXPERIMENTALES}

Para este propósito se han seleccionado dos muestras de maíz morado en mazorca, una de Huánuco y otra de Cañete. Las pruebas o ensayos realizados están orientadas a la molienda fina de los granos de maíz, y a la obtención de soluciones acuosas del principio colorante o antocianicas en base a marlo molido o triturado y mazorca entera o panoja.

\section{MÉTODOS Y TÉCNICAS UTILIZADAS (2)}

\section{Preparación de soluciones}

Las muestras seleccionadas se han codificado de acuerdo a su procedencia: H para Huánuco y $\mathrm{C}$ para Cañete. En base a la composición en masa de las mazorcas de $20 \%$ de marlo y $80 \%$ de granos, todas las soluciones se formulan con 6 litros de agua y 1 kilogramo de mazorcas o $200 \mathrm{~g}$ de marlo triturado. En el siguiente cuadro se detallan las soluciones preparadas para el análisis colorimétrico, que es el promedio de tres pruebas realizadas.
El tiempo de inicio de ebullición de $6 \mathrm{~L}$ de agua es 1 hora, por lo tanto el tiempo de hervido neto de la solución es la diferencia respecto del tiempo total.

\section{Molienda de los granos de maíz}

Los granos obtenidos se procesan en un molino de martillo, obteniéndose harina fina y afrecho.

\section{RESULTADOS EXPERIMENTALES}

El análisis de las soluciones antocianinicas se ha realizado en la Unidad de servicios de Análisis Químicos (USAQ), de la Facultad de Química e Ingeniería Química, utilizando el método colorimétrico. El modelo cromático utilizado es el CIELAB (CIEL*a*b*), usado normalmente para describir todos los colores que puede percibir el ojo humano. Fue desarrollado específicamente con este propósito por la Comisión Internationale d'Eclairage (Comisión Internacional de lluminación), razón por la cual se abrevia "CIE". Los asteriscos $\left({ }^{*}\right)$ que siguen a cada letra forman parte del nombre, representando $L^{*}, a^{*}$ y b*, a "L", "a" y "b", respectivamente.

Cuadro 2. Preparación de soluciones

\begin{tabular}{|c|c|c|c|}
\hline Muestras & Estado & Tiempo hervido $(\mathbf{h})$ & Solución obtenida (L) \\
\hline H1 & Mazorca & 2.5 & 3.5 \\
H2 & Marlo triturado & 1.5 & 4.7 \\
H3 & Mazorca & 1.5 & 4.5 \\
C2 & Mazorca & 2.5 & 3.5 \\
C1 & Marlo triturado & 1.5 & 4.7 \\
\hline
\end{tabular}

Fuente: Elaboración propia. 
Los tres parámetros en el modelo representan la luminosidad de color de acuerdo al siguiente esquema.

\begin{tabular}{|c|c|c|c|}
\hline Parámetro & Rango de color & Valor o ponderación & Color \\
\hline & & 0 & Negro \\
& & 100 & Blanco \\
$\mathrm{L}^{*}$ & Negro-Blanco & + & Verde \\
$\mathrm{a}^{*}$ & Rojo - Verde & - & Amarillo \\
$\mathrm{b}^{*}$ & Amarillo-Azul & + & Azul \\
& & - & \\
\hline
\end{tabular}

El modelo de color CIELAB es tridimensional y solamente puede ser representado adecuadamente en un espacio tridimensional.

En la Figura 1 se puede observar las tendencias de los colores correspondientes a los parámetros a y b.

Las Figuras 2, 3, 4, 5 y 6 muestran los resultados de las pruebas colorimétricas de las soluciones acuosas de maíz morado (panoja) y marlo, considerando los parámetros $L^{*}, a^{*} y b^{*}$. Los resultados obtenidos según el modelo CIELAB toma en cuenta la inclusión o exclusión del componente especular o de transparencia. SCl significa componente especular incluido (con luz blanca) y SCE significa componente especular excluido (sin luz blanca).

En lo referente a la molienda de granos y trituración de marlos, los resultados obtenidos prácticamente no difieren para las dos muestras.

Los cuadros siguientes muestran los resultados unificados obtenidos.

Figura 1. Rango de colores de los parametros "cielab"

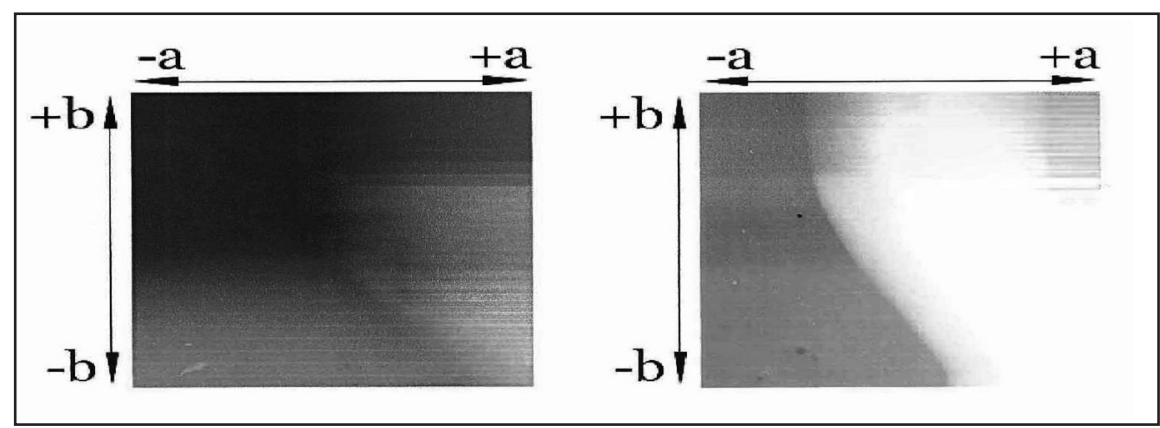

Fuente: USAQ, FQIQ - UNMSM.

Cuadro 3. Molienda de granos de maíz morado

\begin{tabular}{|c|c|c|c|c|}
\hline Granos $(\mathrm{kg})$ & Harina $(\mathrm{kg})$ & Afrecho $(\mathrm{kg})$ & Perdida $(\mathrm{kg})$ & Rendimiento $(\%)$ \\
\hline 20.00 & 17.78 & 1.54 & 0.68 & 96.60 \\
\hline
\end{tabular}

Fuente: Elaboración propia.

Cuadro 4. Trituración de marlos

\begin{tabular}{|c|c|c|c|}
\hline Marlo entero $(\mathrm{kg})$ & Marlo triturado $(\mathrm{kg})$ & Perdida $(\mathrm{kg})$ & Rendimiento $(\%)$ \\
\hline 5.00 & 4.68 & 0.32 & 93.60 \\
\hline
\end{tabular}

Fuente: Elaboración propia. 
EL MAIZ MORADO COMO MATERIA PRIMA INDUSTRIAL

Resultados de pruebas colorimétricas (Figuras N. ${ }^{\circ} 2,3,4,5$ y 6)

\section{FIGURA 2}

\begin{tabular}{|c|c|c|}
\hline Nombre muestra & \multicolumn{2}{|c|}{$\mathrm{H1}$} \\
\cline { 2 - 3 } & $\mathrm{SCl}$ & $\mathrm{SCE}$ \\
\hline $\mathrm{L}^{*}$ & 0.71 & 0.09 \\
\hline $\mathrm{a}^{*}$ & 1.47 & 0.39 \\
\hline$b^{*}$ & -1.73 & -0.55 \\
\hline
\end{tabular}
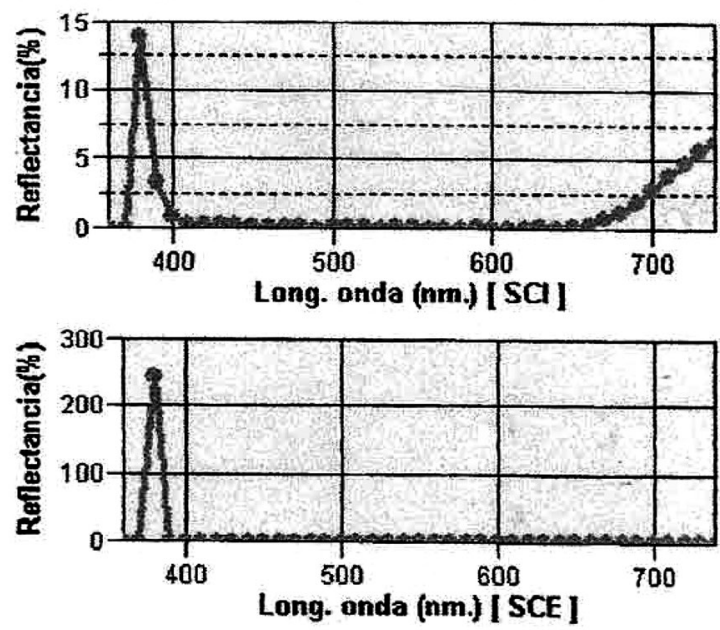

FIGURA 3

\begin{tabular}{|c|c|c|}
\hline Nombre muestra & \multicolumn{2}{|c|}{$\mathrm{H} 2$} \\
\cline { 2 - 3 } & $\mathrm{SCl}$ & $\mathrm{SCE}$ \\
\hline $\mathrm{L}^{*}$ & 12.78 & 0.09 \\
\hline $\mathrm{a}^{*}$ & 7.36 & 0.50 \\
\hline $\mathrm{b}^{*}$ & -0.92 & -0.67 \\
\hline
\end{tabular}
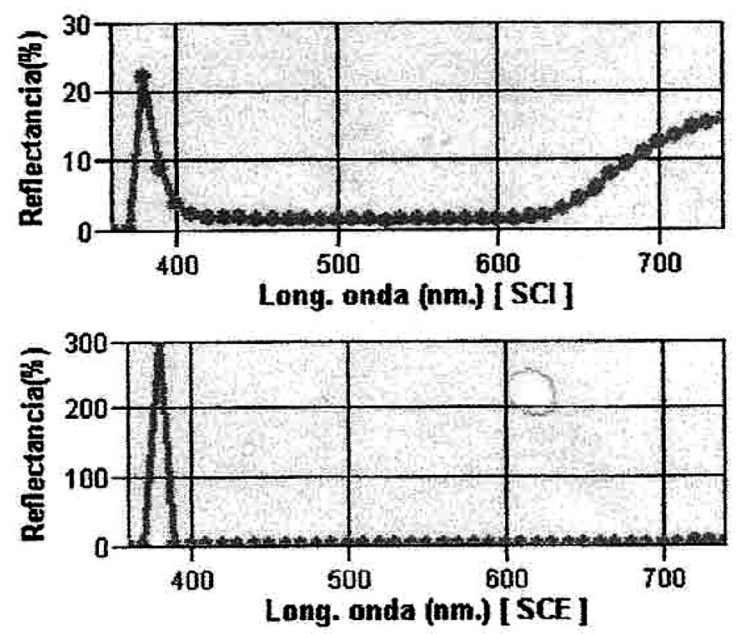
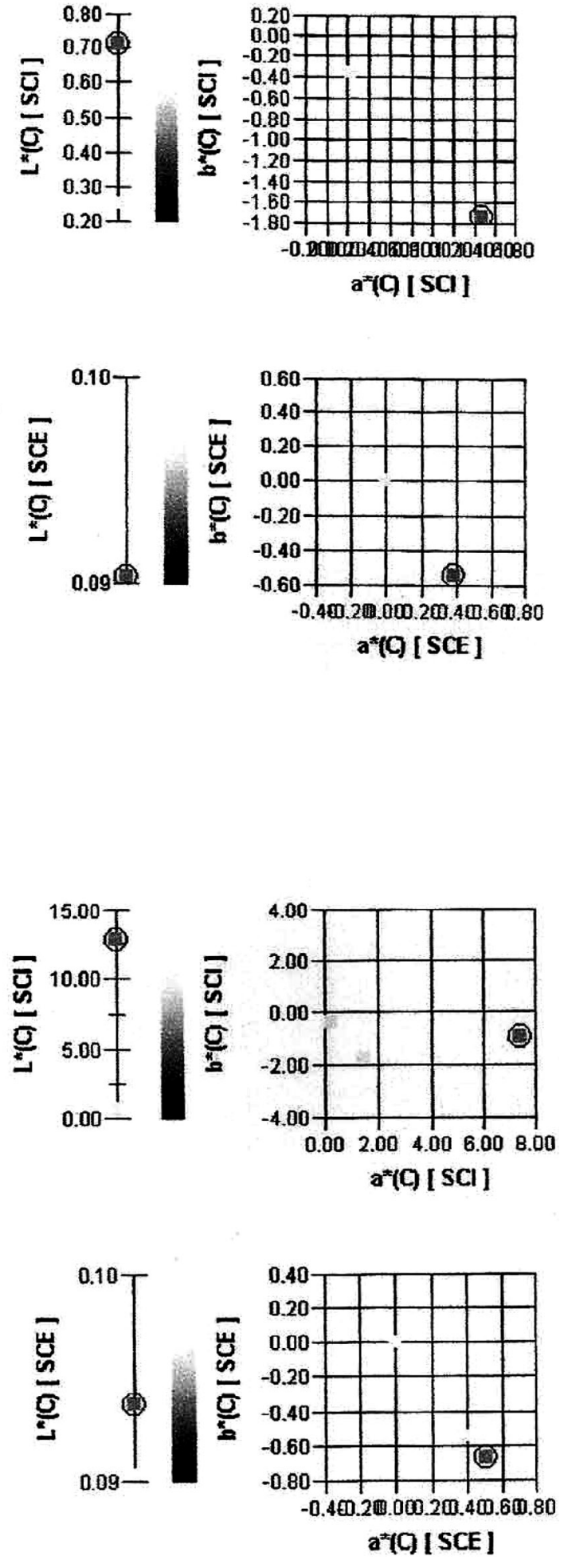
FIGURA 4

\begin{tabular}{|c|c|c|}
\hline Nombre muestra & \multicolumn{2}{|c|}{ H3 } \\
\hline & $5 \mathrm{SC}$ & $\mathrm{SCE}$ \\
\hline & 1.30 & 0.09 \\
\hline $\mathrm{L}^{\wedge}$ & 5.59 & 0.49 \\
\hline $\mathrm{a}^{\mathrm{A}}$ & -0.94 & 0.67 \\
\hline $\mathrm{b}^{\wedge}$ & & \\
\hline
\end{tabular}
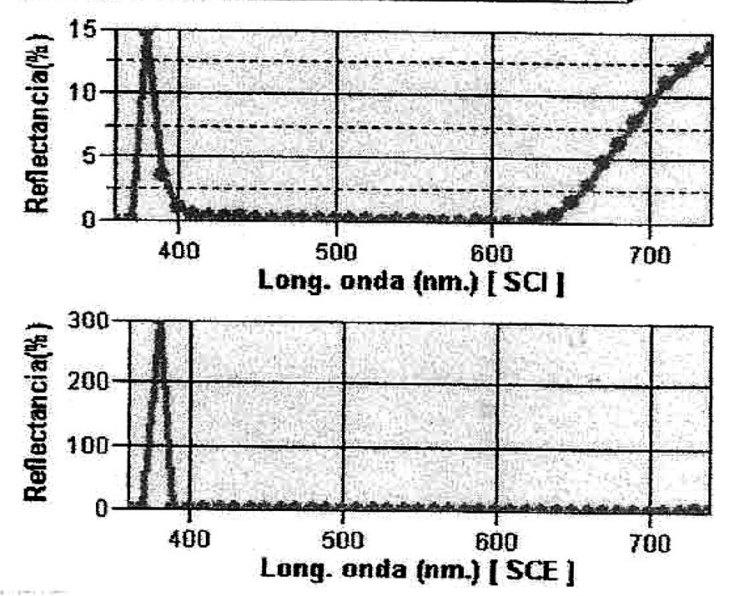

FIGURA 5

\begin{tabular}{|c|c|c|}
\hline Nambre muestra & \multicolumn{2}{|c|}{$\mathrm{C1}$} \\
\cline { 2 - 3 } & $\mathrm{SCl}$ & $\mathrm{SCE}$ \\
\hline & 2.36 & 0.09 \\
\hline $\mathrm{L}^{*}$ & 13.11 & 0.45 \\
\hline $\mathrm{a}^{*}$ & 1.10 & -0.58 \\
\hline $\mathrm{b}^{*}$ & & \\
\hline
\end{tabular}
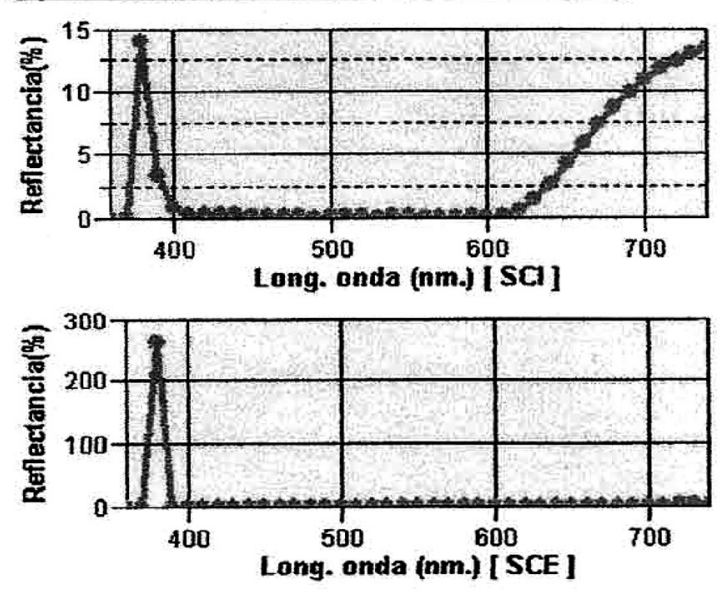
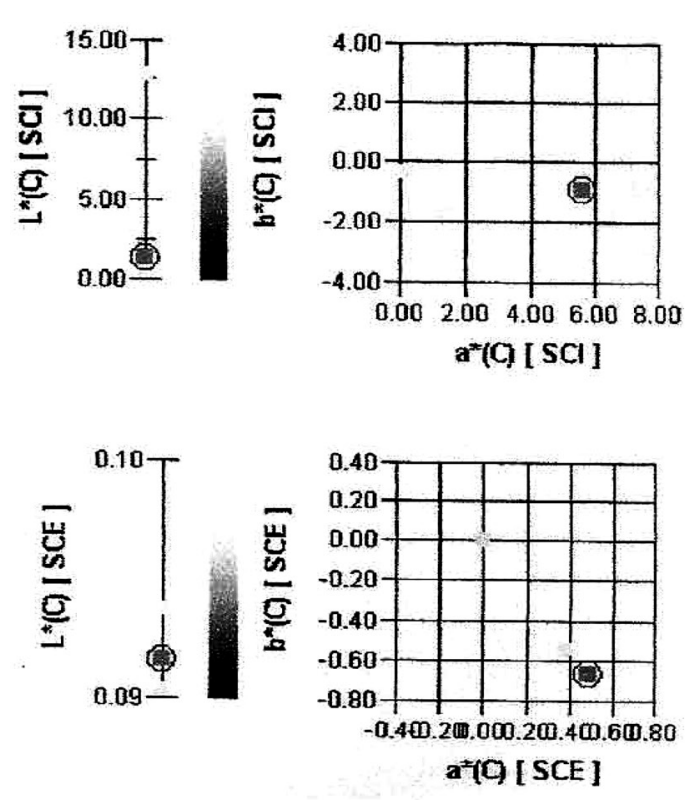
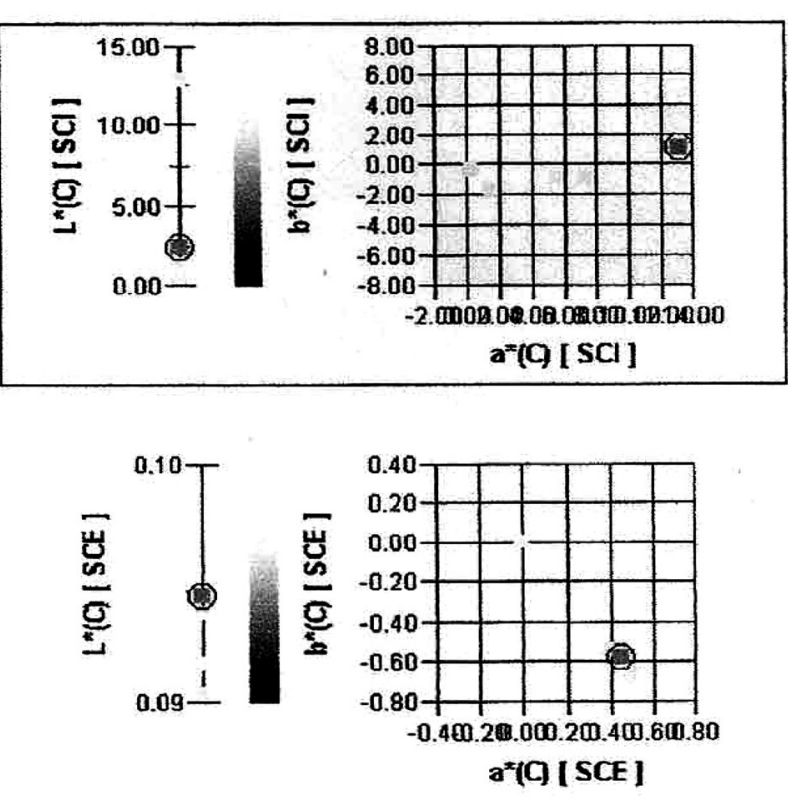


\section{FIGURA 6}

\begin{tabular}{|c|c|c|}
\hline Nombre muestra & \multicolumn{2}{|c|}{$\mathrm{C2}$} \\
\cline { 2 - 3 } & \multicolumn{1}{|c|}{$5 \mathrm{SCl}$} & $\mathrm{SCE}$ \\
\hline & 1.63 & 0.09 \\
\hline $\mathrm{L}^{*}$ & 7.85 & 0.49 \\
\hline $\mathrm{a}^{*}$ & -0.33 & -0.67 \\
\hline $\mathrm{b}^{ \pm}$ & \\
\hline
\end{tabular}
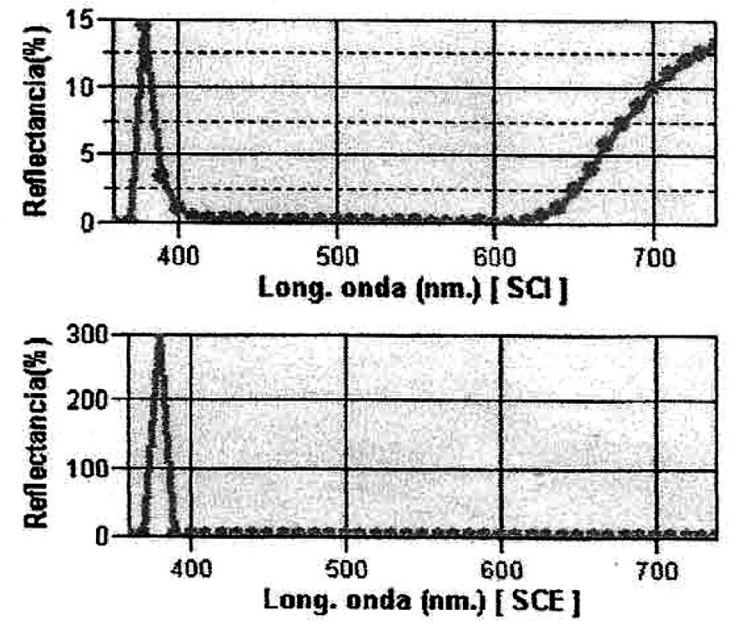

Fuente: USAQ, FQIQ - UNMSM.

\section{INTERPRETACIÓN Y DISCUSIÓN DE RESULTADOS}

- El análisis o interpretación y la discusión de los resultados obtenidos corresponde fundamentalmente a las soluciones anticianinicas.

- En el análisis del Cuadro N. ${ }^{\circ} 2$ se observa que el tiempo de hervido de las muestras con marlo triturado es 1 hora menos que el de las realizadas con mazorca. La muestra $\mathrm{H} 3$ ha sido exprofesamente preparada para 90 minutos de hervido. Las concentraciones iniciales de las soluciones son $0.167 \mathrm{~kg} / \mathrm{L}$ para las muestras con mazorca y $0.033 \mathrm{~kg} / \mathrm{L}$ para las muestras con marlo triturado. La solución obtenida con marlo triturado respecto a la correspondiente con mazorca es mayor en $1.2 \mathrm{~L}$ con excepción de la muestra H3 con un volumen mayor en $200 \mathrm{~mL}$.

- Los resultados obtenidos mediante el modelo CIELAB, que considera los parámetros $L^{*}, a^{*} y b^{*}, y$ la inclusión y exclusión del componente especular son analizados en los párrafos siguientes.

- Para todas las muestras los resultados SCE del parámetro $L^{*}$ son iguales a 0.09 , debido precisamente a la exclusión de la luz blanca.
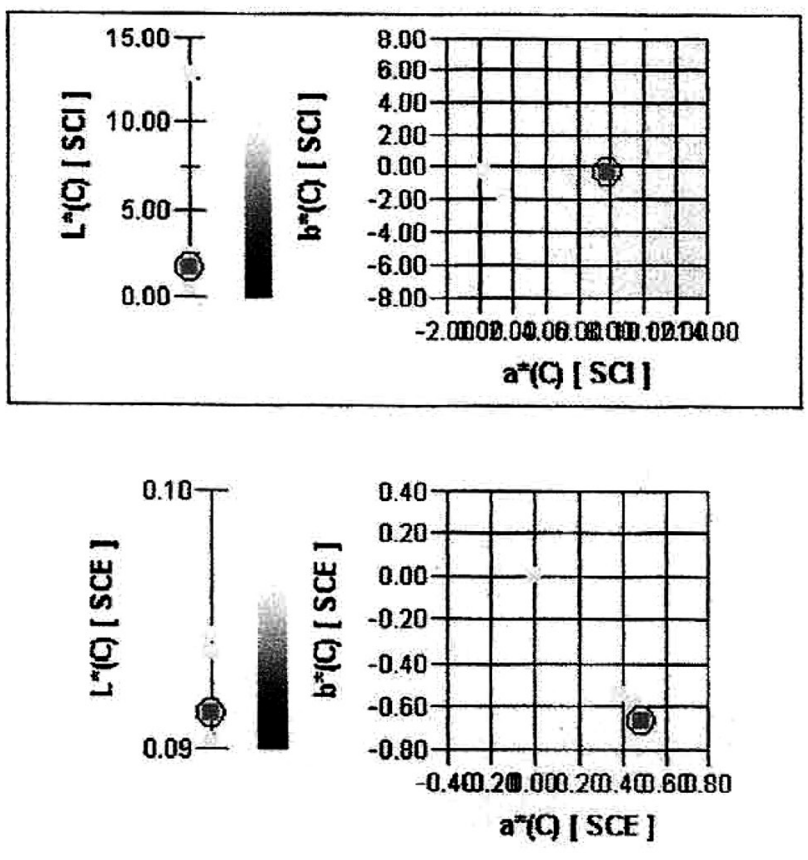

- Los resultados $\mathrm{SCl}$ obtenidos para el parámetro $L^{*}$ son mayores en las soluciones obtenidas con marlo triturado, para las muestras de Huánuco y de Cañete. Este alejamiento del valor cero de $L^{*}$ se explica en razón de que las concentraciones iniciales son menores de las que corresponde a las preparadas con mazorca $(0.033<0.167)$. En todos los casos las muestras $\mathrm{H}$ presentan un mayor valor del parámetro $L^{*}$, en soluciones con mazorca. El valor más alto de $L^{*}$ de la muestra $\mathrm{H} 1$ respecto de $\mathrm{C} 1$, podría explicarse en razón de que el pericarpio de los granos de maíz de Huánuco son más intensos.

- Los resultados SCI y SCE del parámetro a* positivos de las muestras $\mathrm{H}$ muestran una mayor coloración roja en la solución con marlo triturado (H2). En el caso de las muestras C esta tendencia es similar a la anterior.

- Los resultados SCI y SCE del parámetro b* negativos de las muestras $\mathrm{H}$ indican un viraje hacia coloración azul. En cuanto a la muestra $\mathrm{C}$ se observa una distorsión en $\mathrm{C} 1$ con $\mathrm{b}^{*}$ positivo, constituyendo un viraje hacia la coloración amarilla. 


\section{CONCLUSIONES}

- Los altos rendimientos experimentales obtenidos con las muestras, en la producción de harina y marlo triturado constituyen un buen índice para considerar el maíz morado como materia prima industrial, cuyos productos finales pueden ser harina, marlo triturado y afrecho.

- El parámetro L* que es un índice de acercamiento o alejamiento de la transparencia de la solución antocianínica, para fines prácticos constituye una medida para definir la capacidad de coloración del maíz morado por región de origen.

- Haciendo una comparación conjunta de los resultados obtenidos para los parámetros $L^{*}, a^{*}$ y $b^{*}$, la muestra de Cañete presenta una ligera ventaja en soluciones con marlo triturado.

- En ambas muestras, la valoración conjunta de los parámetros que dan valores ligeramente superiores para muestras de soluciones preparadas con mazorcas, se debe a que la concentración de la solución obtenida es mayor. Esto se manifiesta en el volumen menor de la solución obtenida.

- Los gráficos de reflectancia (Figuras N. ${ }^{\circ} 2,3,4$, 5 y 6 ), en función de la longitud de onda adjuntos a los de los parámetros $L^{*}, a^{*}$ y $b^{*}$, constituyen una alternativa de análisis de los resultados obtenidos, similar al de los realizados mediante la Figura N. ${ }^{\circ} 1$.

- En vista de que las diferencias en los valores obtenidas de los parámetros no son significativos, tomando en cuenta el tiempo de hervido y el volumen de la solución obtenida, resulta ventajoso utilizar marlo triturado para preparar chicha y mazamorra morada.

- Debido al uso diferenciado de los granos y el marlo, la harina y el afrecho producidos constituyen una evidente ventaja económica, frente al uso tradicional. Además, la harina producida puede reemplazar a la fécula de papa utilizada en la preparación de mazamorra morada.

\section{RECOMENDACIONES}

- El producto debería continuar mediante la elaboración de un perfil que considere la obtención de harina, marlo triturado, afrecho, chicha morada envasada y gelatina morada, todos ellos productos naturales.

- Habiéndose realizado ensayos de laboratorio de cultivo de maíz morado, fertilizado con abono orgánico líquido (biol), la intensidad de la coloración de la antocianina parece ser más intensa, en consecuencia se deben hacer más ensayos al respecto.

\section{REFERENCIAS BIBLIOGRÁFICAS}

[1] Antonio Manrique Chávez (1995). Maíz Morado Peruano. Instituto Nacional de Investigación Agraria.

[2] Peter Fellows (2007). Tecnología del Procesado de los Alimentos. 2. ${ }^{\circ}$ ed. Editorial Acribia, S. A., Zaragoza. 